\title{
Correction to: Factors associated with late presentation of cervical cancer cases at a district hospital: a retrospective study
}

Priscilla Dunyo ${ }^{1}$, Kofi Effah ${ }^{2}$ and Emilia Asuquo Udofia ${ }^{3^{*}}$

\section{Correction to BMC Public Health (2018) 18:1156 DOI: 10.1186/s12889-018-6065-6}

It has been highlighted, that the original article [1] contained errors in Fig. 1. On the 3rd level, the first box should read "Records with any required information $=157$ " and the second box should read "Records without any required information $=0$ ". Those were incorrectly captured as "Records with complete information $=157$ " and "Records without any required information $=157$ " respectively in the original article. This Correction article shows the correct Fig. 1.

\section{Author details}

'Department of Population, Family and Reproductive Health, School of Public Health, University of Ghana, Legon, Accra, Ghana. ${ }^{2}$ Obstetric and Gynecological Department/Cervical Cancer Screening and Training Center, Catholic Hospital, Battor, Ghana. ${ }^{3}$ Department of Community Health, School of Public Health, University of Ghana, Legon, Accra, Ghana.

Received: 17 October 2018 Accepted: 17 October 2018

Published online: 07 November 2018

\section{Reference}

1. Dunyo P, et al. Factors associated with late presentation of cervical cancer cases at a district hospital: a retrospective study. BMC Public Health. 2018;18: 1156. https://doi.org/10.1186/s12889-018-6065-6.

\footnotetext{
* Correspondence: emiliaudf@yahoo.com

${ }^{3}$ Department of Community Health, School of Public Health, University of

Ghana, Legon, Accra, Ghana
} 


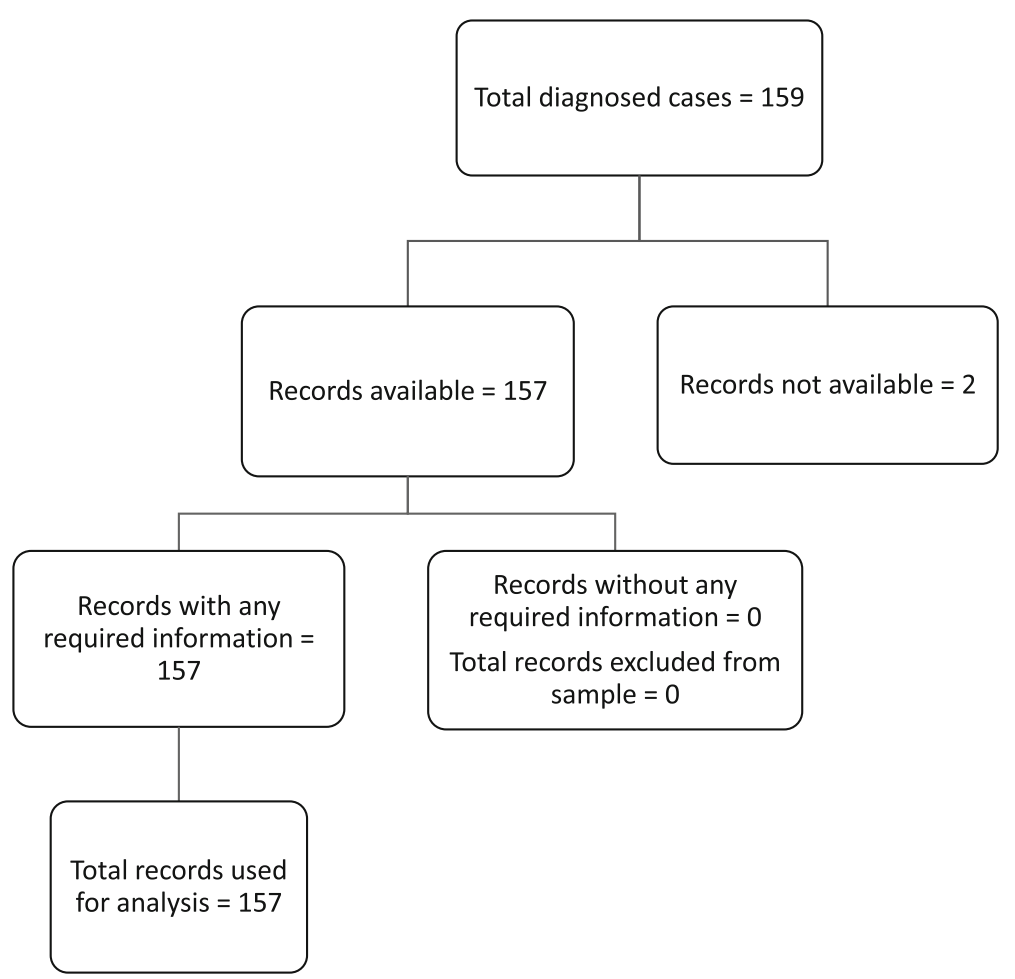

Fig. 1 Schematic diagram showing the record selection process 